\title{
Adrenal Leiomyoma: A Rare Cause of Adrenal Incidentaloma
}

Maria Manuel Costa ${ }^{1,2,3}$, Sandra Belo ${ }^{1,2,3}$, Pedro Souteiro1, João Magalhães ${ }^{4}$ Davide Carvalho ${ }^{1,2,3}$

${ }^{1}$ Department of Endocrinology, Diabetes and Metabolism, Centro Hospitalar São João, Porto, Portugal; ${ }^{2}$ Faculty of Medicine, University of Porto, Porto, Portugal 3 Instituto de Investigação e Inovação da Saúde da Universidade do Porto, 4 Pathology, Centro Hospitalar São João, Porto, Portugal

\section{INTRODUCTION}

- Leiomyomas are benign tumors originating from the smooth muscle cells. They occur more frequently in the uterus and in the gastrointestinal system.

- Adrenal leiomyomas are rare tumors arising from the the smooth muscle of the adrenal vein and its tributaries.

- There are few cases described in the literature.

\section{CASE REPORT}

Identification: 72 year-old man

Date of $\mathbf{1}^{\text {st }}$ consult: April 2013

Presented to our consult with a history of left adrenal incidentaloma detected in abdominal-pelvic CT performed for the study of splenomegaly

Past Medical History: Arterial hypertension

Therapy: Pantoprazole 4omg, Lisinopril 2omg, Amlodipine $5 \mathrm{mg}$

Family history: Irrelevant

Physical examination: Without signs suggestive of hyperfunctioning incidentaloma; Weight $70 \mathrm{Kg}$, Height $1.73 \mathrm{~m}, \mathrm{BMI} 23.4 \mathrm{Kg} / \mathrm{m}^{2}$

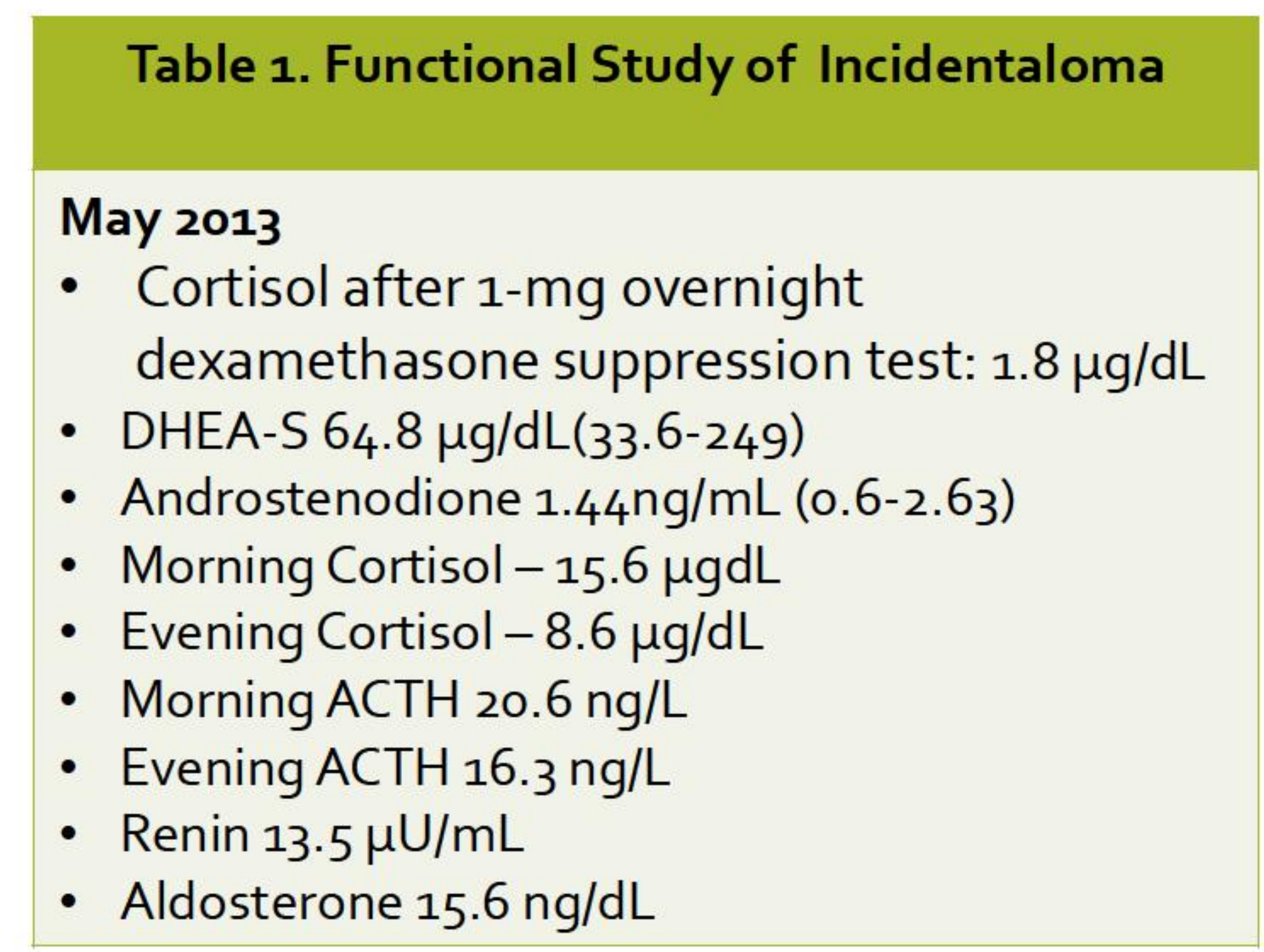

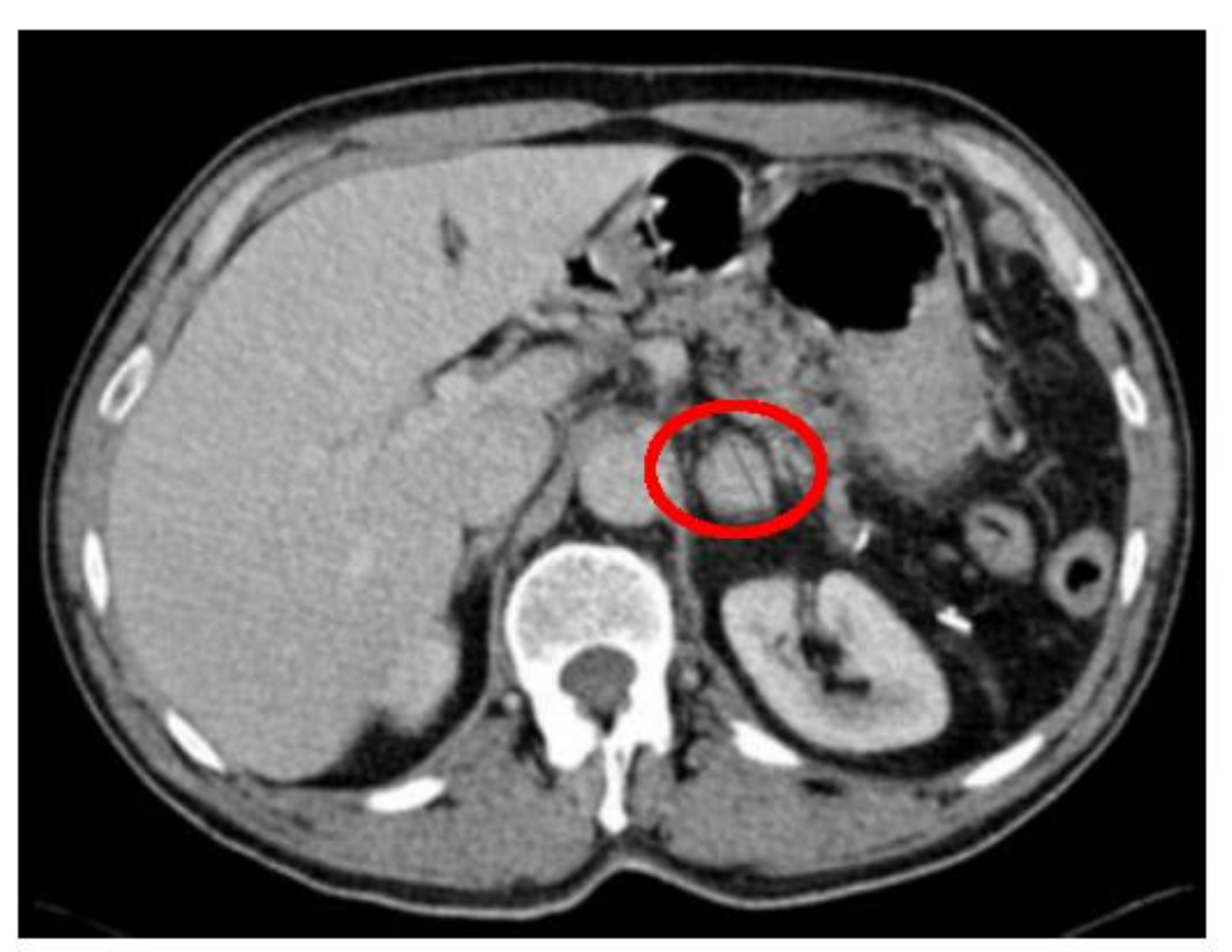

Fig. 2. CT may/2013: Left adrenal nodule with $25 \mathrm{~mm}$, with density values compatible with atypical adenoma

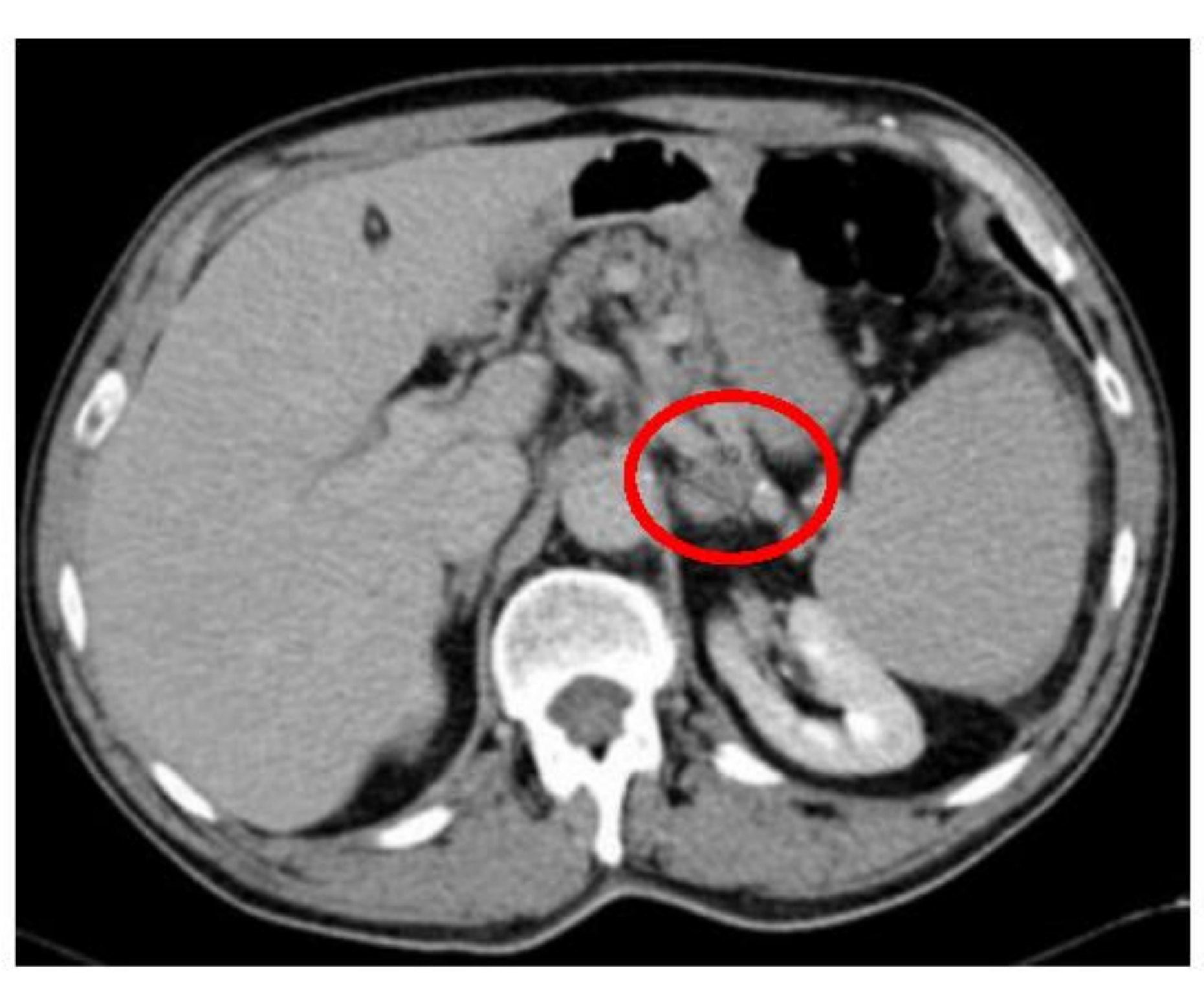

Fig.1. Abdominal-pelvic CT may/2012: Left adrenal incidentaloma with $20 \mathrm{~mm}$ of maximum diameter

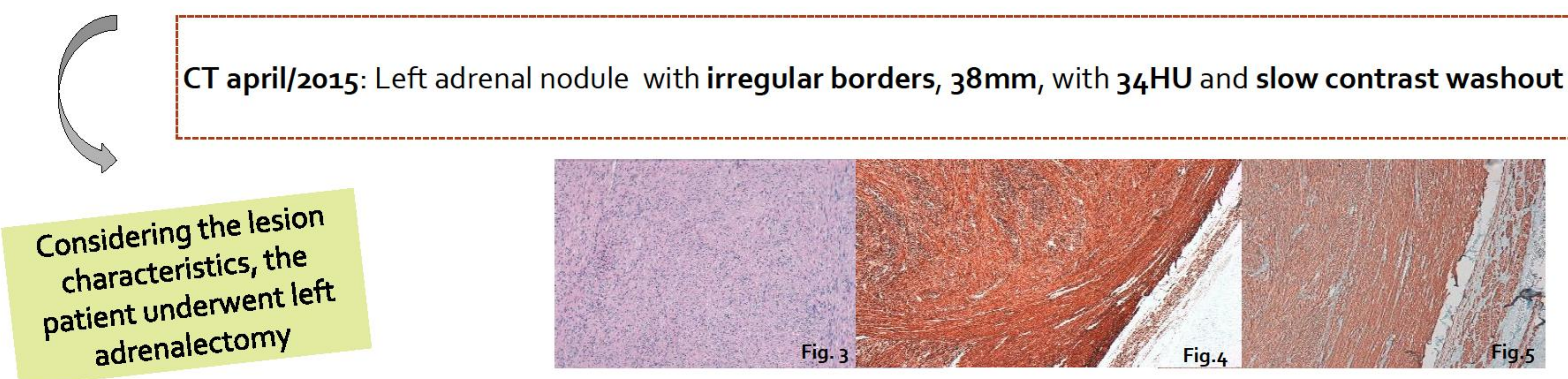

Clinical pathology report: Well-differentiated smooth muscle neoplasm with leiomyoma characteristics (fig. 3). Immunohistochemical study confirmed the diagnosis of leiomyoma with positive cells for alpha-actin (fig. 4) and desmin (fig. 5) and negative for c-Kit and S10o protein.

\section{CONCLUSIONS}

- The adrenal incidentaloma are often found during the investigation of other diseases not related to adrenal and may be a variety of tumours.

- The leiomyoma is very rare and should be considered in the differential diagnosis of incidentalomas.

- Surgical removal is the curative treatment of this benign condition. 